\title{
Tunneling, conductance, and wavevector filtering through magnetic barriers in bilayer graphene
}

\author{
M. Ramezani Masir, ${ }^{1}$ P. Vasilopoulos, ${ }^{2, *}$ and F. M. Peeters ${ }^{1,3, \dagger}$ \\ ${ }^{1}$ Departement Fysica, Universiteit Antwerpen, Groenenborgerlaan 171, B-2020 Antwerpen, Belgium \\ ${ }^{2}$ Department of Physics, Concordia University, Montreal, Quebec, Canada H3G 1 M8 \\ ${ }^{3}$ Departamento de Física, Universidade Federal do Ceará, Caixa Postal 6030, Campus do Pici, 60455-760 Fortaleza, Ceará, Brazil
}

(Received 4 September 2008; published 14 January 2009)

\begin{abstract}
We evaluate the transmission and conductance through magnetic barrier structures in bilayer graphene. In particular we consider a magnetic step, single and double barriers, $\delta$-function barriers, as well as barrier structures that have average magnetic field equal to zero. The transmission depends strongly on the direction of the incident electron or hole wavevector and gives the possibility to construct a direction-dependent wavevector filter. The results contrast sharply with previous results on single-layer graphene. In general, the angular range of perfect transmission becomes drastically wider and the gaps narrower. This perfect transmission range decreases with the number of barriers, the barrier width, and the magnetic field. Depending on the structure, a variety of transmission resonances occur that are reflected in the conductance through the structure.
\end{abstract}

DOI: 10.1103/PhysRevB.79.035409

PACS number(s): 71.10.Pm, 73.21.-b, 81.05.Uw

\section{INTRODUCTION}

Recently the study of single-layer, bilayer, and multilayer graphene has been intensified. The reason is that graphene's electronic properties are drastically different from those, say, of conventional semiconductors. Charge carriers in a wide single-layer graphene behave like "relativistic" chiral massless particles with a "light speed" equal to the Fermi velocity and possess a gapless linear spectrum close to the $K$ and $K^{\prime}$ points. ${ }^{1-3}$ Another consequence is that single-layer and equally bilayer graphene display an unconventional quantum Hall effect. ${ }^{4,5}$ One major consequence is the perfect transmission through arbitrarily high and wide barriers, referred to as Klein tunneling. ${ }^{6-9}$ In addition, the submicron long meanfree paths ${ }^{1}$ will have important consequences for the design of graphene-based devices.

In contrast to carriers in single-layer graphene, those in bilayer graphene possess a quadratic spectrum near the $K$ points and show no Klein tunneling. ${ }^{7}$ Adsorbates and/or gate potentials induce an energy gap due to the tunnel coupling between the layers that is more appropriate for certain applications, e.g., for improving the on/off ratio in carbon-based transistors. A recent review of the properties of graphene is given in Ref. 10.

In a previous paper we studied the transmission through magnetic barrier structures and its angular confinement in single-layer graphene. ${ }^{11}$ In doing so we extended significantly earlier limited results ${ }^{12}$ for a barrier and a step by considering double barriers, $\delta$-function barriers, and barrier structures with inhomogeneous magnetic field profiles which have an average magnetic field of zero that can be realized using nanostructured ferromagnetic strips that are positioned above the graphene layer. We also contrasted the results for electrons with those obtained from the Schrödinger equation. ${ }^{13-15}$ The aim of the present work is to extend our previous study on tunneling through various magnetic barrier structures to bilayer graphene (see Fig. 1 for the layout of the system and Fig. 2 for different magnetic field profiles that can be produced) and critically contrast them with those for single-layer graphene. ${ }^{11,12,16,17}$ As will be seen, the tunnel coupling between the layers significantly modifies some results and further supports bilayer graphene as a promising material for carbon-based devices. The paper is organized as follows. In Sec. II we present the basic formalism. In Sec. III, we present the transmission and conductance results for a magnetic step and single or double barriers and for barrier structures with zero average magnetic field. The limit of $\delta$-function magnetic barriers will also be given for illustrative purposes. We make concluding remarks in Sec. IV.

\section{BASIC FORMALISM}

\section{A. Homogeneous magnetic field}

Before we consider transport in the presence of inhomogeneous magnetic fields, we present the energy levels and corresponding wave functions in a constant magnetic field. Consider a homogeneous magnetic field $B_{0}$ normal to the two-dimensional (2D) plane $(x, y)$ of bilayer graphene. To study transmission through one-dimensional (1D) magnetic barriers we use the Landau gauge for the vector potential $\mathbf{A}(x)=\left(0, B_{0} x, 0\right)$ and make the change $\mathbf{p} \rightarrow \mathbf{p}+e \mathbf{A}$, where $\mathbf{p}$ is the momentum operator. The one-electron Hamiltonian for a graphene bilayer is

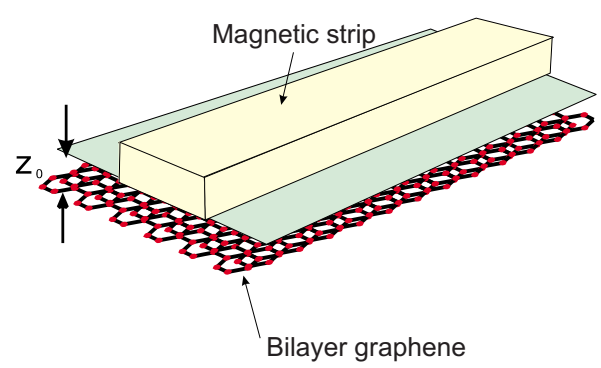

FIG. 1. (Color online) Layout of the system: a ferromagnetic stripe on top of a bilayer graphene sheet separated by a thin oxide layer. 

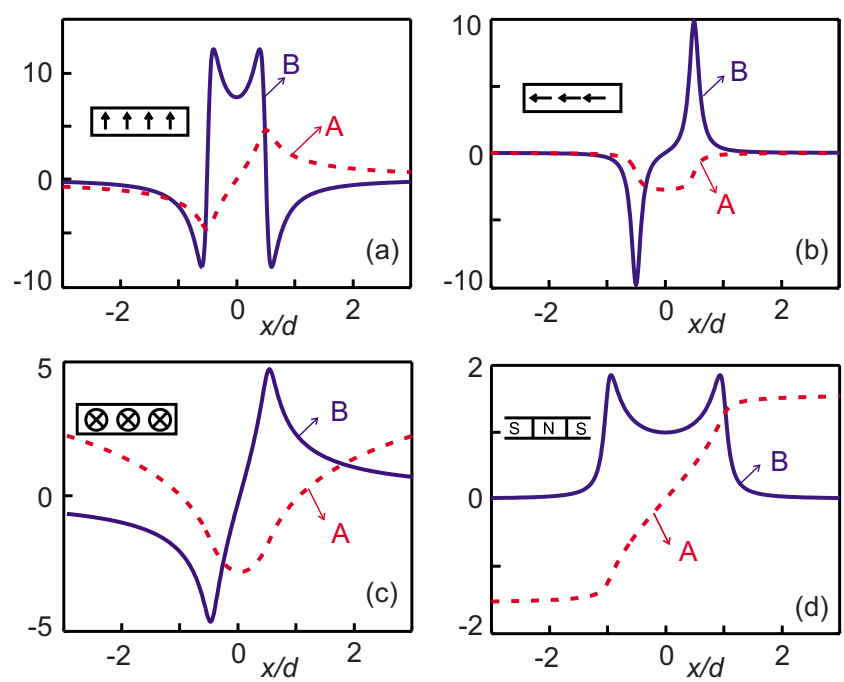

FIG. 2. (Color online) Magnetic field and corresponding vector potential at a distance $z_{0}=0.1$ under the stripe (see Fig. 1) for four different configurations. The stripe is magnetized perpendicularly to its plane in (a) and parallel to it in (b). The profiles in (c) are for a current carrying metallic stripe and those in (d) for a superconducting film containing a normal stripe.

$$
H=\left(\begin{array}{cccc}
V_{1} & \Pi & t & 0 \\
\Pi^{\dagger} & V_{1} & 0 & 0 \\
t & 0 & V_{2} & \Pi^{\dagger} \\
0 & 0 & \Pi & V_{2}
\end{array}\right)
$$

with $\Pi=v_{F}\left[p_{x}+i\left(p_{y}+e A\right)\right]$, where $v_{F}=1 \times 10^{6} \mathrm{~m} / \mathrm{s}$ is the Fermi velocity, $V_{1}$ and $V_{2}$ are the potentials at the two layers, and $t$ is the tunnel coupling between the layers assumed to be constant. This Hamiltonian is valid near the Dirac point $K$ or $K^{\prime}$. Thus scattering between the $K$ and $K^{\prime}$ valleys is neglected. This scattering was shown ${ }^{18}$ to be negligible for fields below $10^{4} \mathrm{~T}$ in single-layer graphene; we expect this to be the case in bilayer graphene as well. It also neglects the real spin of the electrons. However, for the fields of interest here, $B_{0} \leq 1 \mathrm{~T}$, the Zeeman energy is of the order of $10^{-1} \mathrm{meV}$, i.e., negligible compared to the Fermi energy $E_{F} \sim 10 \mathrm{meV}^{3}$ For more details as well as the neglect of trigonal warping appropriate for high energies, see Ref. 19.

To simplify the notation we introduce the length scale $\ell_{B}=\left[\hbar / e B_{0}\right]^{1 / 2}$ and the energy scale $E_{0}=\hbar v_{F} / \ell_{B}$. This allows us to define the following dimensionless quantities:

$$
\begin{gathered}
B(x) \rightarrow B_{0} B(x), \quad A(x) \rightarrow B_{0} \ell_{B} A(x), \quad \vec{r} \rightarrow \ell_{B} \vec{r}, \\
\vec{v} \rightarrow v_{F} \vec{v}, \quad E \rightarrow E_{0} E, \quad t \rightarrow E_{0} t^{\prime} .
\end{gathered}
$$

The Hamiltonian commutes with $p_{y}$ and therefore is a conserved quantity. This allows us to write $\Psi(x, y)=\Phi(x) \exp \left(i k_{y} y\right)$ and solve the equation $H \Psi(x, y)$ $=E \Psi(x, y)$ for the wave function $\Psi(x, y)$ $=\left(\phi_{a}(x), \phi_{b}(x), \phi_{c}(x), \phi_{d}(x)\right)^{T} \exp \left(i k_{y} y\right)$ with $T$ denoting the transpose. Then the components of $\Psi(x, y)$ obey the following coupled differential equations:

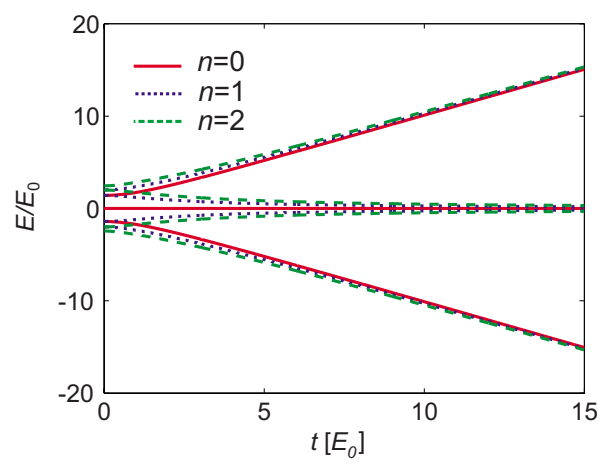

FIG. 3. (Color online) Energy spectrum of a graphene bilayer as a function of $t$ for a homogeneous magnetic field $B=1 \mathrm{~T}$. The single-layer result is obtained for $t=0$.

$$
\left\{\begin{array}{l}
-i\left[d / d x-\left(k_{y}+x\right)\right] \phi_{b}+t^{\prime} \phi_{c}=\left(E-V_{1}\right) \phi_{a}, \\
-i\left[d / d x+\left(k_{y}+x\right)\right] \phi_{a}=\left(E-V_{1}\right) \phi_{b}, \\
-i\left[d / d x+\left(k_{y}+x\right)\right] \phi_{d}+t^{\prime} \phi_{a}=\left(E-V_{2}\right) \phi_{c}, \\
-i\left[d / d x-\left(k_{y}+x\right)\right] \phi_{c}=\left(E-V_{2}\right) \phi_{d} .
\end{array}\right.
$$

Setting $\quad V_{0}=\left(V_{1}+V_{2}\right) / 2, \quad \Delta V=V_{1}-V_{2}, \quad \delta=\Delta V / 2, \quad$ and $\epsilon=E-V_{0}$, Eq. (2) can be decoupled by eliminating the unknowns one at a time. The result for $\phi_{a}$ is

$$
\left[d^{2} / d z^{2}-z^{2} / 4+\gamma_{ \pm} / 2\right] \phi_{a}=0,
$$

where $\quad \gamma_{ \pm}=\epsilon^{2}+\delta^{2} \pm\left[(1-2 \delta \epsilon)^{2}+\left(\epsilon^{2}-\delta^{2}\right) t^{\prime 2}\right]^{1 / 2} \quad$ and $\sqrt{2}\left(x+k_{y}\right)=z$. The solutions of Eq. (3) can be written in terms of the Weber functions. For an asymptotically vanishing wave function for $z \rightarrow \infty$ we define $\phi_{a}(z)=e^{-z^{2} / 4} g(z)$ and substitute it in Eq. (3). For $\delta=0$ and using standard power-series procedures we complete the solution and find the energy spectrum

$$
\epsilon_{n, \pm}= \pm\left\{2 n+1+\frac{t^{\prime 2}}{2} \pm\left[\frac{t^{\prime 4}}{4}+(2 n+1) t^{\prime 2}+1\right]^{1 / 2}\right\}^{1 / 2}
$$

where $n$ is an integer, the Landau-level index. Notice the similarity with the spectrum for single-layer graphene, $E_{n}= \pm \sqrt{2(n+1)}$ or $E_{n}= \pm \sqrt{2 n}$, and the difference from that for the usual electrons with parabolic energy-momentum relation $E_{n}=\hbar \omega_{c}(n+1 / 2)$ consisting of equidistant Landau levels. For $t \rightarrow 0$, Eq. (4) reduces to that of two uncoupled layers with spectrum $E_{n}= \pm \sqrt{2 n+1 \pm 1}$. The spectrum for the first three lowest levels is shown in Fig. 3 as a function of the strength $t$ of the interlayer coupling for a constant magnetic field $B=1$ T. The usual value $t=400 \mathrm{meV}$ corresponds to $t^{\prime}=15$. Notice that varying $t^{\prime}$ for fixed $t$ is equivalent to changing the magnetic field because $E_{0} \sim \sqrt{B}$. In the rest of the paper we fix $t=400 \mathrm{meV}$.

The solution of Eq. (3) can be also written in terms of the well-known Hermite polynomials $H_{n}(x)$. However, this solution is not well suited for regions with discontinuities in the magnetic field that we study here. For this reason we will use Weber functions which are more appropriate. The various wave function components, up to a normalization constant, are 


$$
\Phi^{ \pm}(z) \sim\left(\begin{array}{l}
D\left(p^{ \pm}, z\right) \\
\left(-i \sqrt{2} p^{ \pm} / \epsilon\right) D\left(p^{ \pm}-1, z\right) \\
\left(\epsilon / t^{\prime}-2 p^{ \pm} / t \epsilon\right) D\left(p^{ \pm} z\right) \\
(i \sqrt{2} / \epsilon)\left(\epsilon / t^{\prime}-2 p^{ \pm} / t^{\prime} \epsilon\right) D\left(p^{ \pm}+1, z\right)
\end{array}\right),
$$

where $p^{ \pm}=\left(\gamma_{ \pm}-1\right) / 2$. In regions where the magnetic field is zero and for constant potentials $V_{1}$ and $V_{2}$ with $\delta=0$ we introduce the right-moving solutions

$$
\Psi_{\epsilon, \pm}^{R}(x, y)=N_{ \pm}\left[\mp \epsilon, \mp k_{x}^{ \pm} \pm i k_{y}, \epsilon, k_{x}^{ \pm}+i k_{y}\right]^{T} f^{+}
$$

and the left-moving ones $\left[N_{ \pm}=\left(4 L_{y} \epsilon k_{x}^{ \pm}\right)^{-1 / 2}\right]$

$$
\Psi_{\epsilon, \pm}^{L}(x, y)=N_{ \pm}\left[\mp \epsilon, \pm k_{x}^{ \pm} \pm i k_{y}, \epsilon,-k_{x}^{ \pm}+i k_{y}\right]^{T} f^{-}
$$

with $f^{ \pm} \equiv f^{ \pm}(x, y)=e^{ \pm i k_{x}^{ \pm} x+i k_{y} y}$ and $L_{y}$ being the length of the structure along the $y$ direction. The corresponding energy spectrum and wavevector are

$$
\begin{gathered}
\epsilon=\mp t^{\prime} / 2 \pm\left[t^{\prime 2} / 4+K^{ \pm}\right]^{1 / 2}, \\
k_{x}^{ \pm}=\left[\epsilon^{2}-k_{y}^{2} \pm \epsilon t^{\prime}\right]^{1 / 2},
\end{gathered}
$$

where $K^{ \pm}=k_{x}^{2 \pm}+k_{y}^{2}$. The normalization factor $N_{ \pm}$is obtained from the requirement of unit current carried by each state in the positive or negative $x$ direction,

$$
I=e v \int_{0}^{W} d y \Psi^{\dagger}\left(\begin{array}{cc}
\sigma_{x} & 0 \\
0 & \sigma_{x}
\end{array}\right) \Psi .
$$

\section{B. Conductance}

We will also calculate the conductance $G$ for various magnetic barrier structures by introducing it as the electron flow averaged over half the Fermi surface. ${ }^{14}$ We evaluate $G$ from the standard expression for the total current density $J$, from left to right, given by

$$
J=-e \int_{0}^{\infty} d E \int_{-\pi / 2}^{\pi / 2} T(E, \phi)\left[f_{l}(E)-f_{r}(E)\right] v_{x}(E) \rho(E) E d \phi .
$$

Here $f_{l}(E)\left(f_{r}(E)\right)$ is the Fermi-Dirac function on the left (right), $\rho(E)$ is the density of states, and $T(E, \phi)$ is the transmission as a function of the energy $E$ and the angle of incidence $\phi$ relative to the $x$ direction. In the linear transport regime and for low temperatures we can replace $f_{l}-f_{r}$ by a $\delta$ function and obtain the conductance

$$
G=G_{0} \int_{-\pi / 2}^{\pi / 2} T\left(E_{F}, E_{F} \sin \phi\right) \cos \phi d \phi,
$$

with $G_{0}=\left(2 e^{2} / h\right)\left(L_{y} / \pi \hbar v_{F}\right)\left[E_{F}^{2}+t E_{F}\right]^{1 / 2}$ and $E_{F}$ is the Fermi energy.

\section{MAGNETIC FIELD STEP AND SINGLE AND DOUBLE BARRIERS}

\section{A. Magnetic field step}

We consider a region $x<0$, in which there is no magnetic field, followed by one $x>0$ in which there is a constant magnetic field $B$. This is described by
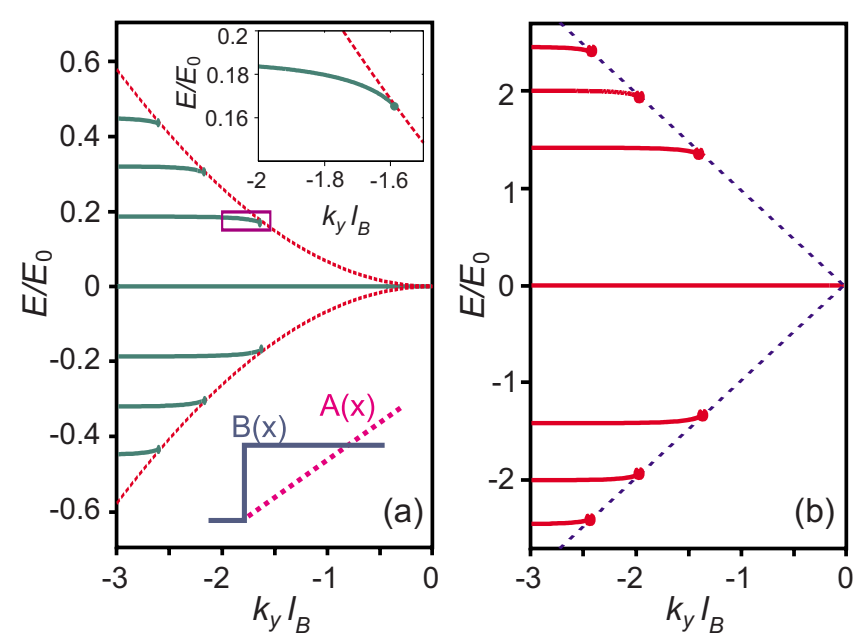

FIG. 4. (Color online) Bound-state energy spectrum vs wavevector component $k_{y}$ parallel to the magnetic step. Panel (a) is for a bilayer step with $t^{\prime}=15$ and panel (b) for a magnetic step in single-layer graphene.

$$
B(x)=B_{0} \Theta(x) .
$$

For $x<0$ the solution is that of a free particle while for $x>0$ the solution is a combination of the solutions for the homogenous magnetic field case, i.e., Eq. (5). The solution for $x<0$ can be written as

$$
\Psi_{\epsilon, k_{x}^{ \pm}}^{ \pm}=e^{ \pm k_{x}^{ \pm} x}\left[1,-\frac{i}{\epsilon}\left( \pm k_{x}^{ \pm}+k_{y}\right), \mp 1, \pm \frac{i}{\epsilon}\left( \pm k_{x}^{ \pm}-k_{y}\right)\right]^{T}
$$

and the full wave function is

$$
\Psi^{\mathrm{I}}=c_{1} \Psi_{\epsilon, k_{x}^{+}}^{+}+c_{2} \Psi_{\epsilon, k_{x}^{-}}^{+}
$$

For $x>0$ the result is

$$
\Psi^{\mathrm{II}}=c_{3} \Phi^{+}(z)+c_{4} \Phi^{-}(z),
$$

with $z=\sqrt{2}\left(x+k_{y}\right)$ and $\Phi^{ \pm}(z)$ given by Eq. (5). Matching the solutions at $x=0$ gives the homogeneous set of algebraic equations in the matrix form

$$
\left(\begin{array}{cccc}
1 & 1 & s^{+} & s^{-} \\
z^{+} & z^{-} & b^{+} & b^{-} \\
a^{+} & a^{-} & f^{+} & f^{-} \\
e^{+} & e^{-} & g^{+} & g^{-}
\end{array}\right)\left(\begin{array}{c}
c_{1} \\
c_{2} \\
c_{3} \\
c_{4}
\end{array}\right)=0
$$

Here $z^{ \pm}=\frac{-i}{E}\left(k_{x}^{ \pm}+k_{y}\right), \quad e^{ \pm}=\frac{-i}{E}\left(k_{x}^{ \pm}-k_{y}\right) a^{ \pm}, \quad s^{ \pm}=D\left(p^{ \pm}, \sqrt{2} k_{y}\right)$, $f^{ \pm}=\left(\frac{E}{t^{\prime}}-\frac{2 p^{ \pm}}{t^{\prime} E}\right) s^{ \pm}, \quad b^{ \pm}=\left(-i \sqrt{2} p^{ \pm} / \epsilon\right) D\left(p^{ \pm}-1, \sqrt{2} k_{y}\right)$, $g^{ \pm}=(i \sqrt{2} / \epsilon)\left(\epsilon / t^{\prime}-2 p^{ \pm} / t^{\prime} \epsilon\right) D\left(p^{ \pm}+1, \sqrt{2} k_{y}\right), \quad$ and $a^{ \pm}$ $=\epsilon / t^{\prime}+\left(k_{x}^{ \pm 2}-k_{y}^{2}\right) / \epsilon t^{\prime}$. The dispersion relation is obtained from the condition $\operatorname{det}(M)=0$. As a function of $k_{y}$ the spectrum is shown in Fig. 4(a). We contrast it with that for a single layer in Fig. 4(b). Both spectra terminate at the corresponding free-electron results with the single-layer straight lines $E=\hbar v_{F} k_{y}$ in (b) replaced with the parabolas $E=\hbar v_{F} k_{y}\left[\mp t^{\prime} / 2 \pm\left(t^{\prime 2} / 4+k_{v}^{2}\right)^{1 / 2}\right]$ in (a). Notice also how different the level spacing is between the two panels. For large 


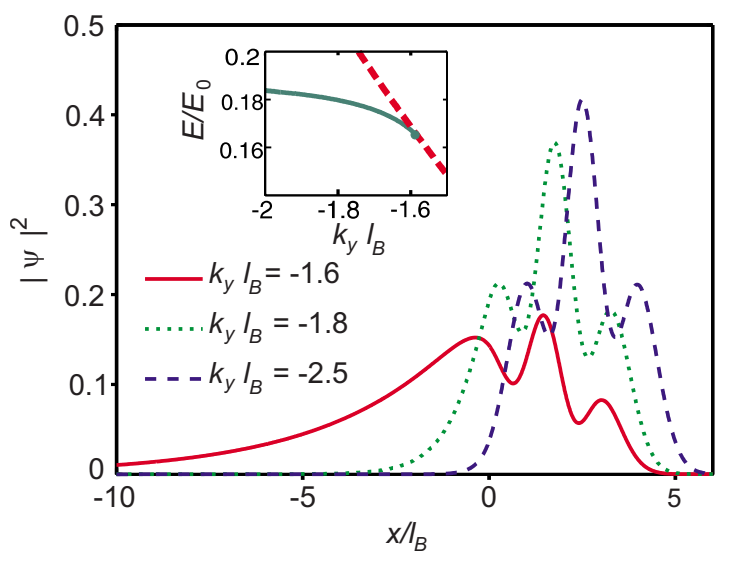

FIG. 5. (Color online) The electron probability density $|\Psi|^{2}=\left|\phi_{a}\right|^{2}+\left|\phi_{b}\right|^{2}+\left|\phi_{c}\right|^{2}+\left|\phi_{d}\right|^{2}$ of the $n=1$ Landau level, in a magnetic step, for different values of $k_{y}$.

negative $k_{y}$ the particles, localized around $x_{0}=-k_{y} l_{B}$, are deep into the magnetic field region and their spectrum is that of the previously mentioned Landau levels. For $k_{y}$ values close to the free-particle branch (dotted curves) the energy spectrum attains a dispersion (with the exception of the $n=0$ or $E=0$ level) implying that the states have a nonzero velocity. Such states are confined in the $x$ direction near the barrier edge (see Fig. 5) and move along it.

\section{B. Single and double barriers}

\section{Energy spectrum}

We consider a magnetic barrier of width $d$ shown in the inset of Fig. 6(a). The corresponding vector potential $\mathbf{A}(x)$ is given by

$$
A(x)=B_{0} \begin{cases}-d / 2, & x<-d / 2 \\ x, & -d / 2<x<d / 2 \\ d / 2, & x>d / 2 .\end{cases}
$$

In region I for $x<-d / 2$ the solution can be written as
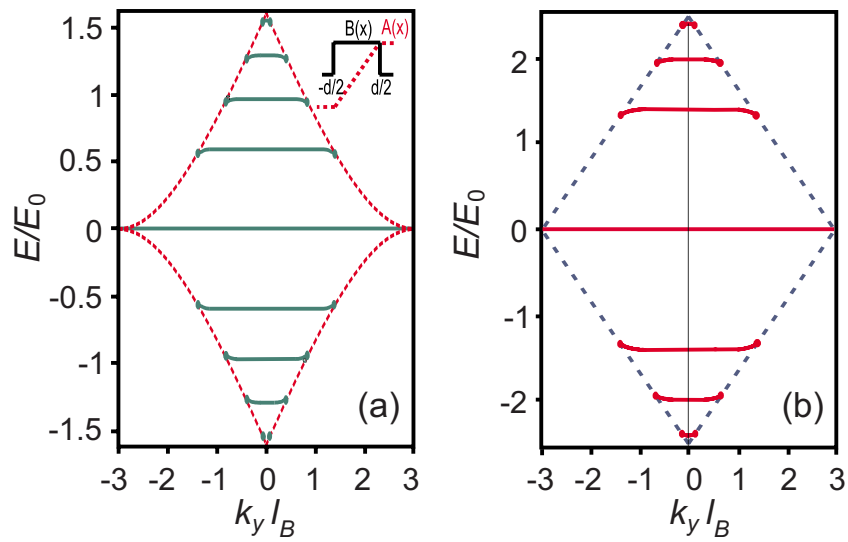

FIG. 6. (Color online) As in Fig. 4 for a magnetic barrier of width $d=3 l_{B}$.

$$
\Psi^{\mathrm{I}}=c_{1} \Psi_{E, k_{x}^{+}}^{+}+c_{2} \Psi_{E, k_{x}^{-}}^{+}
$$

in region II $(-d / 2<x<d / 2)$ as

$$
\Psi^{\mathrm{II}}=f_{1} \Phi^{-}(z)+f_{2} \Phi^{-}(-z)+f_{3} \Phi^{+}(z)+f_{4} \Phi^{+}(-z)
$$

with $z=\sqrt{2}\left(k_{y}+x\right)$, and in region III $(x>d / 2)$ as

$$
\Psi^{\mathrm{III}}=h_{1} \Psi_{E, k_{x}^{+}}^{-}+h_{2} \Psi_{E, k_{x}^{-}}^{-}
$$

The continuity of the wave function at the edges of the barrier $x= \pm d / 2$ and current conservation give

$$
\begin{gathered}
\Psi_{k_{x}^{ \pm}}^{\mathrm{I}}(-d / 2)=\Psi^{\mathrm{II}}(-d / 2), \\
\Psi^{\mathrm{II}}(d / 2)=\Psi_{k_{x}^{ \pm}}^{\mathrm{III}}(d / 2) .
\end{gathered}
$$

From these relations we can connect $\Psi^{\mathrm{III}}(d / 2)$ with $\Psi^{\mathrm{I}}(-d / 2)$ with a matrix. Setting the determinant of this matrix equal to zero gives the dispersion relation. The resulting expressions are rather involved and will not be given here. Numerical results for the dispersion relation are given in Fig. 6(a) and are contrasted with the corresponding ones for a single-layer barrier in Fig. 6(b). As in Fig. 4, we notice here the same difference in level spacing and the bending of the levels near the free-particle result where $k_{y}$ is replaced with $k_{y} \pm d / 2$ (red dashed curves). Notice that in contrast to the magnetic step case, where the spectrum is limited only from the right, it is now limited from the left as well and the pertinent $k_{y}$ values fall, approximately, in the range $-3 \leq k_{y} \ell_{B} \leq 3$.

\section{Transmission}

In contrast to the magnetic step problem, where the transmission is zero for any value of the momentum, in the case of a magnetic barrier of finite width the transmission can attain nonzero values. To evaluate the transmission through a single magnetic barrier we write the solution in the three domains, denoted earlier as I, II, and III, as follows. Region I $(x<-d / 2)$ :

$$
\Psi_{ \pm}^{I}=\Psi_{E, \pm}^{R}+r_{+}^{ \pm} \Psi_{E,+}^{L}+r_{-}^{ \pm} \Psi_{E,-}^{L},
$$

region $\mathrm{II}(-d / 2 \leq x \leq d / 2)$ :

$$
\Psi^{\mathrm{II}}=c_{1} \Phi^{-}(z)+c_{2} \Phi^{-}(-z)+c_{3} \Phi^{+}(z)+c_{4} \Phi^{+}(-z),
$$

and region III $(x>d / 2)$ :

$$
\Psi_{ \pm}^{\mathrm{III}}=t_{+}^{ \pm} \Psi_{E,+}^{R}+t_{-}^{ \pm} \Psi_{E,-}^{R} .
$$

Here $r^{ \pm}$and $t^{ \pm}$are the reflection and transmission coefficients, respectively. After matching the solutions at $x= \pm d / 2$ we obtain the transmission matrix as

$$
t(E, q)=\left(\begin{array}{cc}
t_{+}^{+}\left(E, k_{y}\right) & t_{-}^{+}\left(E, k_{y}\right) \\
t_{+}^{-}\left(E, k_{y}\right) & t_{-}^{-}\left(E, k_{y}\right)
\end{array}\right),
$$

and the transmission probability from

$$
T=\operatorname{Tr}\left(t t^{\dagger}\right) .
$$

A contour plot of the transmission probability is shown in panel (a) of Fig. 7 and is contrasted with that for a single- 

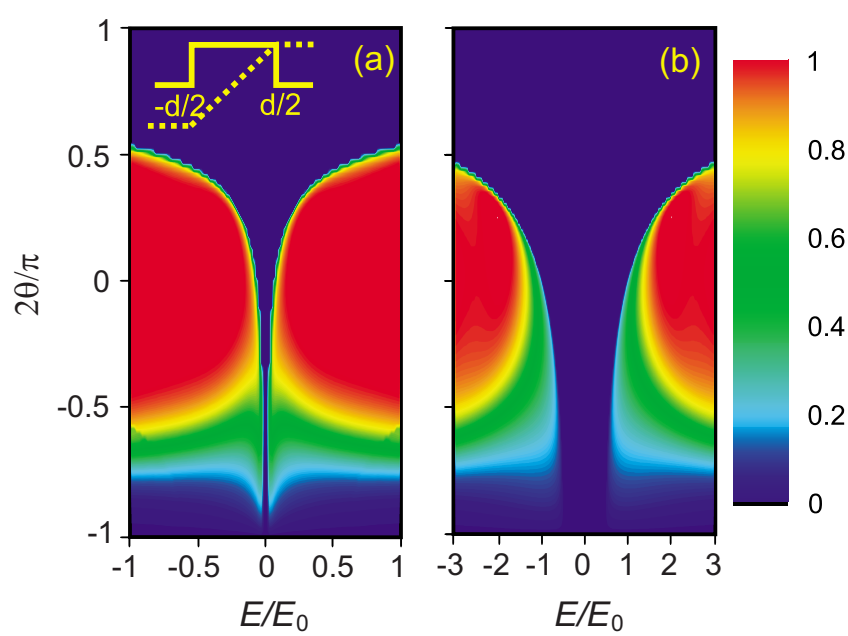

FIG. 7. (Color online) (a) Contour plot of the transmission $T$ through a magnetic barrier with $d=2 l_{B}$. The corresponding result for a single-layer graphene barrier is shown in (b). The angle $\theta$ is measured from the direction of normal incidence.

layer graphene barrier in panel (b). Comparing the two panels we see that in the bilayer case the angular range of perfect transmission becomes wider whereas the energy gap becomes narrower. For real wavevectors $k_{x}$ we can express the angular confinement of the transmission through a bilayer barrier as

$$
-1 \leq \sin \theta^{ \pm} \leq 1-d / \sqrt{E^{2} \pm E t^{\prime}},
$$

where $\theta^{ \pm}=\arctan \left[\left(k_{y}-d / 2\right) / k_{x}^{ \pm}\right]$. In Fig. 7(a) we have $\theta=\theta^{+}$for $E>0$ and $\theta=\theta^{-}$for $E<0$. The corresponding result for a single-layer barrier is obtained from Eq. (28) for $t^{\prime}=0$. If we do not use the dimensionless units, the last term in Eq. (28) is multiplied by $\lambda=\hbar v_{F} / \ell_{B}^{2}$, i.e.,

$$
d / E \rightarrow \lambda d / E, d /\left(E^{2} \pm E t\right)^{1 / 2} \rightarrow \lambda d /\left(E^{2} \pm E t\right)^{1 / 2} .
$$

For a double barrier we proceed in the same manner as for a single one with the vector potential shown in the inset of Fig. 8 and given by

$$
A= \begin{cases}0, & x<-L / 2-d \\ {[x+(L / 2+d)] / d,} & -L / 2-d \leq x \leq-L / 2 \\ 1, & -L / 2<x<L / 2 \\ {[x-(L / 2-d)] / d,} & L / 2 \leq x \leq L / 2+d \\ 2, & x>L / 2+d .\end{cases}
$$

We obtain the transmission probability after matching the solutions at the four interfaces. In Fig. 8(a) we show a contour plot of the transmission, as a function of the angle of incidence and energy, and contrast it with that for the case of graphene in Fig. 8(b). We see again the same similarities and differences between the two panels that we saw in Fig. 7. In addition, upon comparing panel (a) in both figures we see how the gap widens and the angular range of perfect transmission shrinks upon increasing the number of barriers. Thus the allowed range of carriers that are able to transmit through both barriers is smaller than for a single magnetic barrier. Notice also that relative to the single-layer graphene [see Fig.
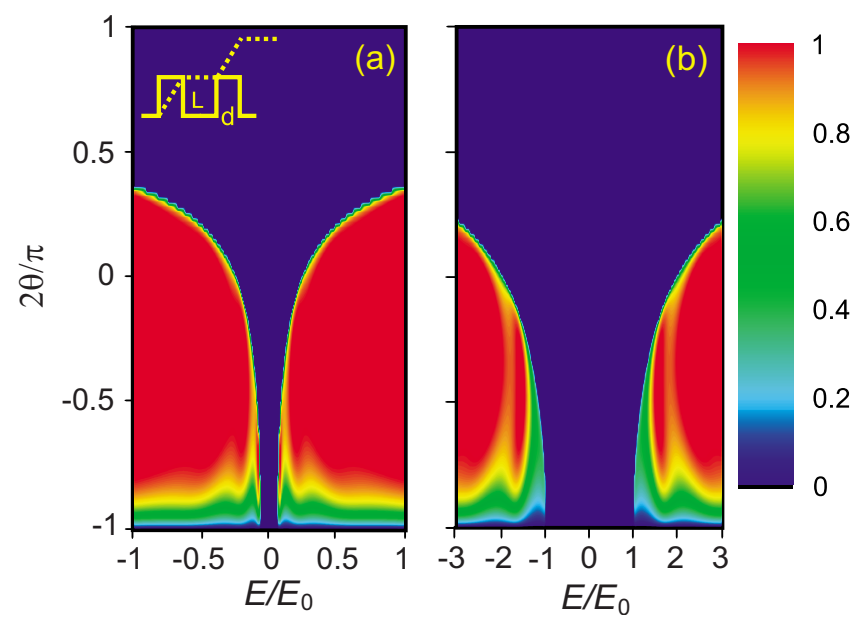

FIG. 8. (Color online) (a) Contour plot of the transmission $T$ through a double magnetic barrier with $d=l_{B}$ and $L=2 l_{B}$. The result for single-layer graphene is shown in (b).

8(b)] we are forced, due to the linear spectrum, to consider wider intervals in energy to see any transmission.

\section{Structures with $\langle B\rangle=0$}

Here we consider magnetic structures with inhomogeneous magnetic field profiles but such that the average magnetic field vanishes, i.e., with $\langle B\rangle=0$, and compare the transmission probability through them with that through the same single-layer structures with linear spectrum. Such magnetic field profiles are typically obtained when we overlay nanostructured ferromagnetic stripes on a graphene bilayer [see Fig. 2(a)]. In Fig. 9(a) we show a contour plot of the transmission probability for the structure shown in its inset for $d=3 l_{B}$ and in Fig. 9(b) the corresponding single-layer result. In this case the transmission probability is symmetric with respect to the angle of incidence and the angular range for $\theta$ is given by
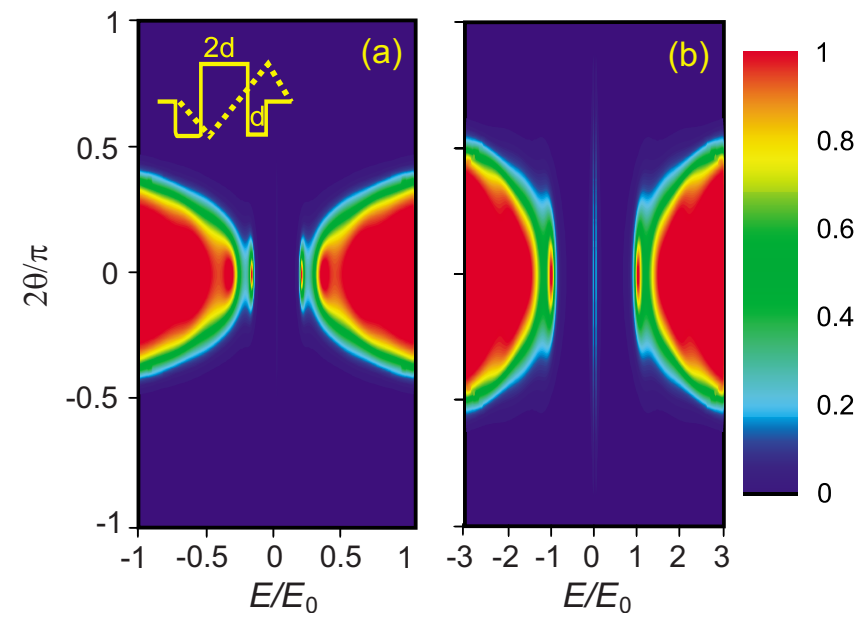

FIG. 9. (Color online) (a) Contour plot of the transmission $T$ through a barrier structure (see inset) with $\langle B\rangle=0$ and $d=1.5 l_{B}$. The result for single-layer graphene is shown in (b). 

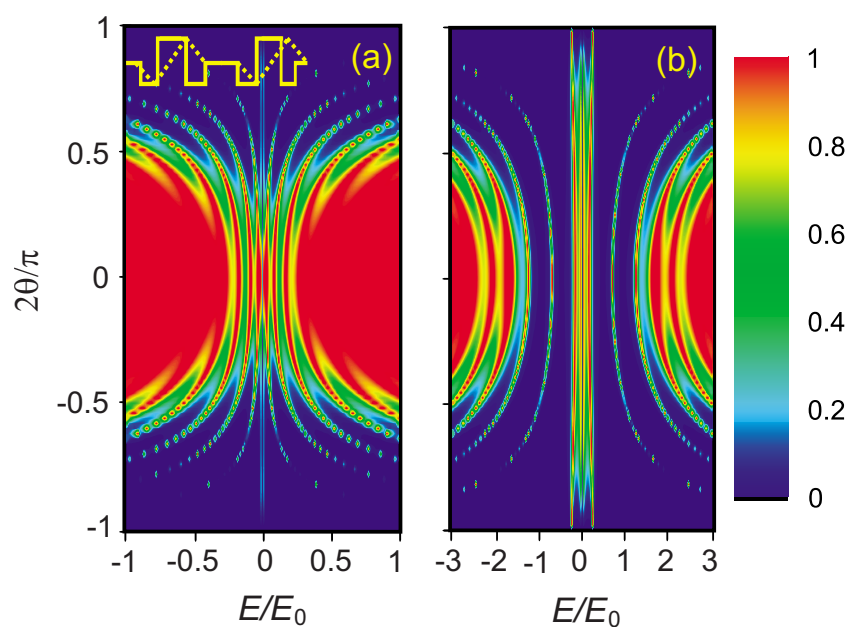

FIG. 10. (Color online) (a) Contour plot of the transmission $T$ through a unit twice that shown in Fig. 9(a) with $d=l_{B}$ and $L=3 l_{B}$. (b) As in (a) for the same structure on single-layer graphene.

$$
-1+d / \sqrt{E^{2} \pm E t^{\prime}} \leq \sin \theta^{ \pm} \leq 1-d / \sqrt{E^{2} \pm E t^{\prime}} .
$$

Beyond this range, there is still a nonzero tunneling because of the evanescent wave solutions inside the magnetic barrier. The result for single-layer graphene is obtained by setting $t^{\prime}=0$ in Eq. (31) and reads

$$
-1+d / E \leq \sin \theta \leq 1-d / E .
$$

Again, Eq. (29) applies if we use the standard units.

In Fig. 10 we show a contour plot of the transmission for a two-unit structure, the unit shown in the inset of Fig. 9(a). Again panel (a) shows the bilayer result and panel (b) the single-layer one. Notice that as compared to the simple double magnetic barrier structure (see Fig. 8) the transmission: (1) is even with respect to the angle of incidence, (2) exhibits a very rich set of resonances, and (3) is mainly nonzero for angles close to perpendicular incidence. The conductance of this double unit is shown in Fig. 11(b) and is contrasted with the one for a single unit [see Fig. 9(a)]. Clear resonances are observed as a function of the Fermi energy of the particles. We compare these results with the corresponding one for the simple double magnetic barrier [see inset of Fig. 8(a)] in Fig. 11(a) for two different interbarrier distances. Only a very weak resonant structure is found when the magnetic barriers are separated over larger distances. Notice also the zero conductance region for small Fermi energy which is absent in Fig. 11(b) and which can be easily understood from the transmission plots (see Fig. 8).

\section{Delta-function magnetic barriers}

It is instructive to consider the limit of delta-function magnetic barriers which considerably simplifies the calculations. For a single magnetic $\delta$-function barrier we have $B(x)=B_{0} l_{B} \delta(x)$; the corresponding vector potential is $A(x)=B_{0} l_{B}[\theta(x)-\theta(-x)] / 2$, where $\theta(x)$ is the step function. Here we have two regions $x<0$ and $x>0$. The solutions for regions I and II are

$$
\Psi_{ \pm}^{I}=\Psi_{E, \pm}^{R}+r_{+}^{ \pm} \Psi_{E,+}^{L}+r_{-}^{ \pm} \Psi_{E,-}^{L},
$$
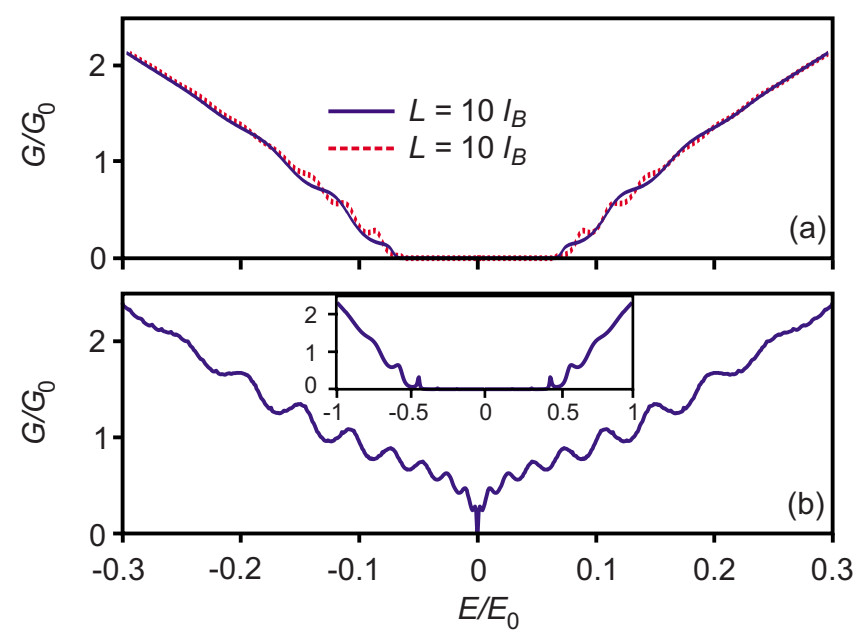

FIG. 11. (Color online) Conductance $G$ as a function of energy through a double magnetic barrier (inset of Fig. 8) with $d=l_{B}$ for two different $L$ 's. (b) $G$ through the structure shown in Fig. 10(a) with $\langle B\rangle=0, d=l_{B}$, and $L=10 l_{B}$. The inset shows $G$ through the structure shown in Fig. 9(a) with $d=3 l_{B}$.

$$
\Psi_{ \pm}^{\mathrm{II}}=t_{+}^{ \pm} \Psi_{E,+}^{R}+t_{-}^{ \pm} \Psi_{E,-}^{R} .
$$

After matching the wave functions at $x=0$, the transmission amplitudes $t_{\bar{\mp}}^{ \pm}$can be combined in the transmission matrix given by Eq. (26); the transmission probability is given by Eq. (27).

For two magnetic $\delta$-function barriers [shown in Fig. $12(\mathrm{a})]$ the corresponding vector potential is

$$
A(x)=B_{0} \ell_{B} \begin{cases}0, & x<-L / 2 \\ 1, & -L / 2<x<L / 2 \\ 0, & x>L / 2 .\end{cases}
$$

We consider three regions I $(x<-L / 2)$, II $(-L / 2<x<L / 2)$, and III $(x>L / 2)$. The three solutions are

$$
\Psi_{ \pm}^{I}=\Psi_{E, \pm}^{R}+r_{+}^{ \pm} \Psi_{E,+}^{L}+r_{-}^{ \pm} \Psi_{E,-}^{L},
$$
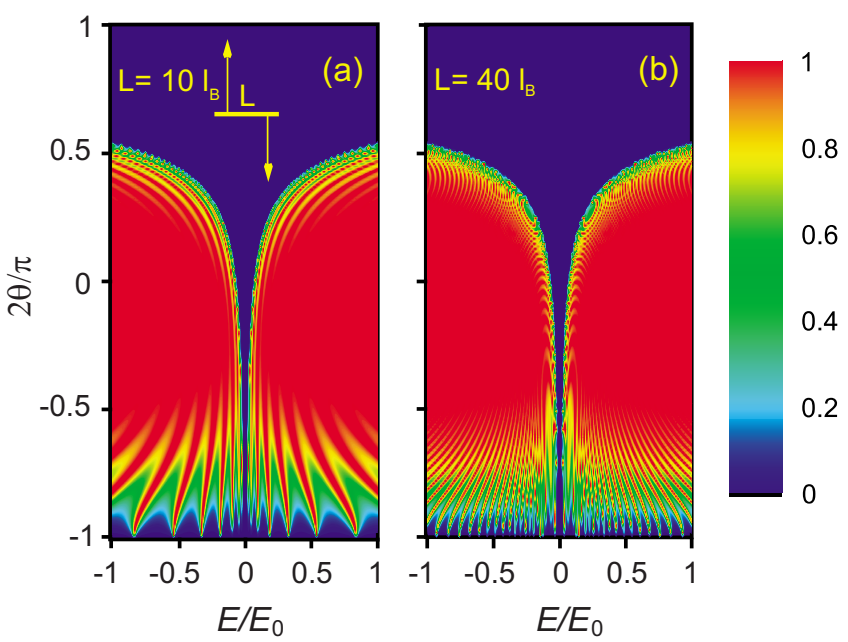

FIG. 12. (Color online) Contour plot of the transmission through two magnetic $\delta$-function barriers, of equal strength but opposite sign, with $t=15 E_{0}$. It is $L=10 \ell_{B}$ in (a) and $L=40 \ell_{B}$ in (b). 

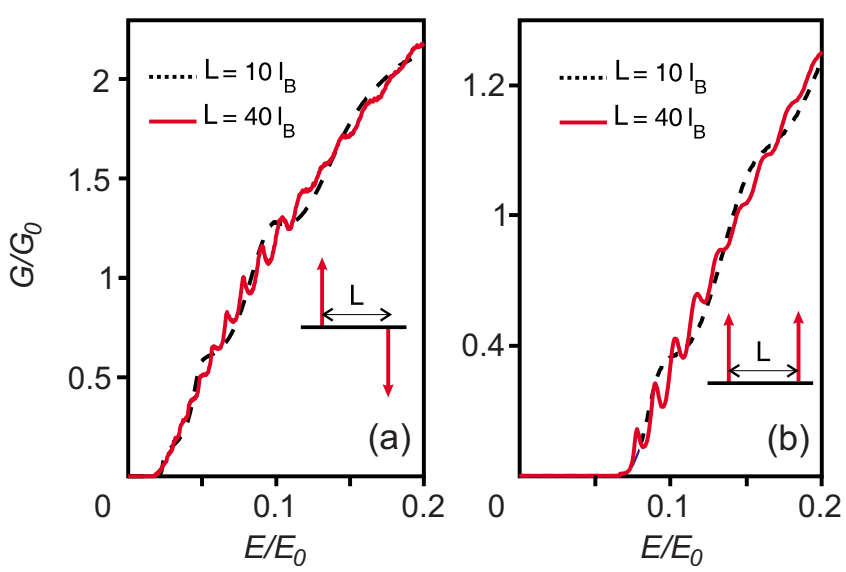

FIG. 13. (Color online) Conductance, as a function of energy, of two magnetic $\delta$-function barriers, of equal strength, in the (a) same or (b) opposite direction.

$$
\Psi_{ \pm}^{\mathrm{II}}=c_{1} \Psi_{E,+}^{R}+c_{2} \Psi_{E,-}^{R}+c_{3} \Psi_{E,+}^{L}+c_{4} \Psi_{E,-}^{L},
$$

and

$$
\Psi_{ \pm}^{\mathrm{III}}=t_{+}^{ \pm} \Psi_{E,+}^{R}+t_{-}^{ \pm} \Psi_{E,-}^{R} .
$$

Here $k_{x}^{\prime \pm}=\left[E^{2}-\left(k_{y}+1\right)^{2} \pm E t^{\prime}\right]^{1 / 2}, k_{x}^{\prime \prime \pm}=k_{x}^{ \pm}$. After matching at $x=-L / 2$ and $x=L / 2$ we can find the transmission matrix elements. Numerical results for the transmission probability are shown in Fig. 12 for two different values of the distance between the $\delta$ functions. We see clearly well-defined resonances for $\theta<\pi / 4$, the number of which increases appreciably with increasing value of $L$. These resonances are a consequence of quantum mechanical interference effects which occur for $k_{x}=k \cos \theta=n \pi / L$ with $E=\hbar v_{F} k$. The conductance through two magnetic $\delta$ functions is shown in Fig. 13. Notice the similarity between Figs. 13 and 11(a). Both show a zero conductance region for small energies which are more pronounced in both $\delta$ functions having the same sign. There are also weak oscillations which are more pronounced when the interbarrier separation is large.

\section{CONCLUDING REMARKS}

We evaluated the transmission through various magnetic barrier nanostructures on bilayer graphene and contrasted it with that in the same structures on single-layer graphene. In particular, we treated a magnetic step, single and double regular or $\delta$-function barriers, as well as complex structures with inhomogeneous magnetic field profiles but such that the average magnetic field vanishes. To demonstrate the main physics we limited ourselves to simple model magnetic field profiles that qualitatively approximate experimentally realizable nonhomogeneous magnetic field profiles.

We showed that the transmission exhibits a strong dependence on the direction of the incident electron or hole wavevector. In general, the angular range of perfect transmission becomes drastically wider and the gaps drastically narrower. This perfect transmission range decreases with increasing number of barriers. Moreover, the transmission through the complex structures shows much more pronounced resonances than that through single or double barriers. An important feature of the transmission results is their dependence on the angle of incidence as shown in several figures and highlighted in Ref. 11 for a single barrier: the transmission is finite only in a certain range of angles of incidence [cf. Eqs. (3), (28), and (31)]. Given the connection between the wavevectors and $\theta$, this is equivalent to wavevector filtering or confinement. One can further modify the angular dependence of the transmission with double barriers [cf. Fig. 8]. The main parameters that control this angular dependence are the width of the barriers/wells, the energy of the incident electrons, and the magnetic field [cf. Eq. (29)]. Given the rapid progress in the field and the quest for carbon-based nanostructure devices, ${ }^{15}$ we expect that the predictions/findings of this paper will be tested experimentally in the near future. We defer to future work the influence of spin and spin-orbit interactions in transport through these or similar graphene nanostructures.

\section{ACKNOWLEDGMENTS}

This work was supported by the Flemish Science Foundation (FWO-Vl), the Belgian Science Policy (IAP), the Brazilian National Research Council CNPq, and the Canadian NSERC Grant No. OGP0121756.

\footnotetext{
*takis@alcor.concordia.ca

†rancois.peeters@ua.ac.be

${ }^{1}$ K. S. Novoselov, A. K. Geim, S. V. Morozov, D. Jiang, Y. Zhang, S. V. Dubonos, I. V. Grigorieva, and A. A. Firsov, Science 306, 666 (2004).

${ }^{2}$ Y. Zhang, Y. W. Tan, H. L. Störmer, and P. Kim, Nature (London) 438, 201 (2005).

${ }^{3}$ Y. Zhang, Z. Jiang, J. P. Small, M. S. Purewal, Y.-W. Tan, M. Fazlollahi, J. D. Chudow, J. A. Jaszczak, H. L. Stormer, and P. Kim, Phys. Rev. Lett. 96, 136806 (2006).

${ }^{4}$ V. P. Gusynin and S. G. Sharapov, Phys. Rev. Lett. 95, 146801
}

(2005).

${ }^{5}$ K. S. Novoselov, A. K. Geim, S. V. Morozov, D. Jiang, and M. I. Katsnelson, Nat. Phys. 2, 177 (2006); E. McCann and V. I. Fal'ko, Phys. Rev. Lett. 96, 086805 (2006).

${ }^{6}$ O. Klein, Z. Phys. 53, 157 (1929).

${ }^{7}$ M. I. Katsnelson, K. S. Novoselov, and A. K. Geim, Nat. Phys. 2, 620 (2006).

${ }^{8}$ J. M. Pereira, Jr., V. Mlinar, F. M. Peeters, and P. Vasilopoulos, Phys. Rev. B 74, 045424 (2006).

${ }^{9}$ J. Milton Pereira, Jr., F. M. Peeters, and P. Vasilopoulos, Appl. Phys. Lett. 90, 132122 (2007). 
${ }^{10}$ A. K. Geim and K. S. Novoselov, Nature Mater. 6, 183 (2007).

${ }^{11}$ M. Ramezani Masir, P. Vasilopoulos, A. Matulis, and F. M. Peeters, Phys. Rev. B 77, 235443 (2008).

${ }^{12}$ A. De Martino, L. Dell' Anna, and R. Egger, Phys. Rev. Lett. 98, 066802 (2007); Solid State Commun. 144, 547 (2007).

${ }^{13}$ F. M. Peeters and A. Matulis, Phys. Rev. B 48, 15166 (1993).

${ }^{14}$ A. Matulis, F. M. Peeters, and P. Vasilopoulos, Phys. Rev. Lett. 72, 1518 (1994).
${ }^{15}$ I. S. Ibrahim and F. M. Peeters, Phys. Rev. B 52, 17321 (1995). ${ }^{16}$ L. Oroszlany, P. Rakyta, A. Kormanyos, C. J. Lambert, and J. Cserti, Phys. Rev. B 77, 081403(R) (2008).

${ }^{17}$ F. Zhai and K. Chang, Phys. Rev. B 77, 113409 (2008).

${ }^{18}$ S. Park and H. S. Sim, Phys. Rev. B 77, 075433 (2008).

${ }^{19}$ A. H. Castro Neto, F. Guinea, N. M. R. Peres, K. S. Novoselov, and A. K. Geim, e-print arXiv:0709.1163v2, Rev. Mod. Phys. (to be published). 